KODAI MATH. SEM. REP.

24 (1972), 142-145

\title{
ON THE MINIMUM MODULUS OF A MEROMORPHIC ALGEBROID FUNCTION OF LOWER ORDER LESS THAN ONE HALF
}

\author{
By KōKICHI SHIBAZAKI
}

1. Ostrovskii [4] has proved the following:

Let $f(z)$ be a meromorphic function of lower order $\lambda$. If $\lambda<1 / 2$, then

$$
\limsup _{r \rightarrow \infty} \frac{\log ^{+} \mu(r, f)}{T(r, f)} \geqq \frac{\pi \lambda}{\sin \pi \lambda}[\cos \pi \lambda-1+\delta(\infty)],
$$

where $\mu(r, f)=\inf \{|f(z)| ;|z|=r\}$ and $\delta(a)$ is the Nevanlinna deficiency of $f(z)$ at $a$.

In this note we shall extend the above theorem to an $n$-valued meromorphic algebroid function of lower order less than one half.

It is well known that for algebroid functions even if a function $y(z)$ is entire and of order zero Wiman's theorem does not always hold on the covering Riemann surface defined by $y(z)$. If, however, we use the minimum modulus of the maximum of the determinations of $y(z)$, then Wiman's theorem for it holds. Recently Ozawa [5] has extended Wiman's theorem of $\cos \pi \lambda$-type ([2]) to an $n$-valued entire algebroid function of lower order less than one.

2. Let $y(z)$ be an $n$-valued meromorphic algebroid and non-algebraic function of lower order $\lambda$ defined by an irreducible equation

$$
F(z, y) \equiv y^{n}+A_{1} y^{n-1}+\cdots+A_{n-1} y+A_{n}=0,
$$

where each $A_{i}(i=1,2, \cdots, n)$ is meromorphic in $|z|<+\infty$ and $n$ is an integer greater than one. Following Ozawa [5] we define the minimum modulus $\mu(r, y)$ of $y(z)$ by $\mu(r, y)=\inf \left\{\max _{1 \leq \jmath \leq n}\left|y_{j}(z)\right| ;|z|=r\right\}$, where $y_{j}$ is the $j$-th determination of $y(z)$.

Then we shall prove the following

THEOREM. If $\lambda<1 / 2$, then

$$
\limsup _{r \rightarrow \infty} \frac{\log ^{+} \mu(r, y)}{T(r, y)} \geqq \frac{\pi \lambda}{\sin \pi \lambda}\left[\frac{1}{k} \cos \pi \lambda-1+\delta(\infty)\right],
$$

where $k$ is the number of coefficients $A_{3}$ transcendental in the defining equation (2.1).

\footnotetext{
Received February 25, 1971.
} 
3. According to Selberg [6] we have the following relation between the coefficients $A_{j}$ in (2.1) and the determinations $y_{j}$ of $y(z)$ :

$$
\log \left|A_{j}\right| \leqq \sum_{i=1}^{n} \log ^{+}\left|y_{i}\right|+{ }_{n} C_{[n / 2]} \quad(j=1,2, \cdots, n) .
$$

Therefore we get

which implies

$$
\log \max _{1 \leqq j \leqq n}\left|A_{j}\right| \leqq n \log \max _{1 \leqq i \leqq n}\left|y_{i}\right|+O(1)
$$

$$
\log \mu(r, A) \leqq n \log ^{+} \mu(r, y)+O(1),
$$

where $A=\max _{1 \leqq \jmath \leqq n}\left|A_{j}\right|$.

Moreover for all transcendental coefficients $A$, we obtain the following inequalities:

$$
\frac{1}{n} N\left(r, \infty, A_{j}\right)-O(\log r) \leqq N(r, \infty, y) \leqq \frac{1}{n} \Sigma N\left(r, \infty, A_{\imath}\right)+O(\log r)
$$

and

$$
\frac{1}{n} T\left(r, A_{j}\right)-O(\log r) \leqq T(r, y) \leqq \frac{1}{n} \Sigma T\left(r, A_{\imath}\right)+O(\log r) .
$$

Here in (3.2), (3.3) and in the sequal each summation $\Sigma$ is taken over all $i$ such that the $A_{2}$ in (2.1) are transcendental. From this last inequality we see that if $y(z)$ is of lower order $\lambda$, then every $A_{\jmath}$ is of lower order at most $\lambda$. The converse is also true.

Denoting the number of transcendental coefficients $A_{j}$ in (2.1) by $k$ we derive from (3.1)

$$
\frac{n \log ^{+} \mu(r, y)}{T(r, y)} \geqq \frac{\log \mu(r, A)+O(1)}{T(r, y)} \geqq \frac{\Sigma \log }{\mu\left(r, A_{j}\right)+O(1)} \frac{}{k T(r, y)} .
$$

4. A lemma. Let $f(z)$ be a meromorphic function of lower order $\lambda, \lambda<1$, with $f(0)=1$. Following Ostrovskii and Goldberg we can construct for $f(z)$

$$
H_{f}(r)=\sum_{\left|a_{i}\right|<R} \log \left(1+\frac{r}{\left|a_{i}\right|}\right)+\sum_{\left|b_{i}\right|<R} \log \left(1+\frac{r}{\left|b_{i}\right|}\right),
$$

where $r<R$ and $\left\{a_{i}\right\}$ and $\left\{b_{i}\right\}$ are zeros and poles of $f(z)$, respectively, and we have $H_{f}(r) \leqq$ const. $T(2 R, f)$ for $r \leqq R$.

Then we have the following

Lemma ([1], [4]). For $0<\xi<\eta<R$ and $0<\sigma<1$

$$
\begin{aligned}
& \int_{\xi}^{\eta}\left\{\log ^{+} \mu(r, f)+\frac{\pi \sigma}{\sin \pi \sigma}[N(r, \infty, f)-\cos \pi \sigma N(r, 0,1 / f)]\right\} \frac{d r}{r^{1+\sigma}} \\
\geqq & C^{\prime} H_{f}(\xi) \xi^{-\sigma}-C^{\prime \prime}(1-\sigma)^{-1} R^{-\sigma} T(2 R, f),
\end{aligned}
$$


where $C^{\prime}$ and $C^{\prime \prime}$ are two positive constants.

5. Proof of Theorem. From our assumption there exist $k$ transcendental coefficients $A_{\jmath}$ in the defining equation (2.1) of $y(z)$. For each $A_{\jmath}$ of such transcendental functions we can choose a value $\alpha_{\jmath}$ satisfying

$$
\lim _{r \rightarrow \infty} \frac{N\left(r, 0, A_{j}-a_{j}\right)}{T\left(r, A_{j}\right)}=1
$$

(for example take $a$, not contained in a set $\{a\}$ of inner capacity zero [3]).

Then we define $\left\{B_{j}(z)\right\}$ as follows. For each $A_{j}$ of $k$ transcendental functions we put

$$
B_{j}(z)=\frac{A_{j}(z)-a_{\jmath}}{c_{0}} \quad \text { if } \quad A_{j}(z)-a_{\jmath}=c_{0}+c_{m} z^{m}+\cdots
$$

where $c_{m} \neq 0$ and $m$ is a non-zero integer, or

$$
B_{j}(z)=\frac{A_{j}(z)-a_{\jmath}}{c_{m^{\prime}}^{\prime} z^{m^{\prime}}} \quad \text { if } \quad A_{j}(z)-a_{\jmath}=c_{m^{\prime}}^{\prime} z^{m^{\prime}}+\cdots,
$$

where $c_{m^{\prime}}^{\prime} \neq 0, c_{0} \neq 0$ and $m^{\prime}$ is a non-zero integer.

From this definition of $B_{j}$ and (5.1) we obtain for each $B_{\jmath}$

$$
\begin{aligned}
& N\left(r, \infty, B_{j}\right)=N\left(r, \infty, A_{j}\right)+O(\log r) \\
& N\left(r, 0, B_{j}\right)=(1+o(1)) T\left(r, A_{j}\right), \\
& \log \mu\left(r, B_{j}\right) \leqq \log \mu\left(r, A_{j}\right)+O(\log r) \quad \text { as } \quad r \rightarrow \infty .
\end{aligned}
$$

Further we have each $B_{j}(0)=1$. Consequently as Ostrovskii [4] did we can construct the function $H_{j}(r)$ in $\S 4$ for each $B_{j}(z)$. We can apply the above Lemma to such functions $B_{j}(z)$. Hence with arbitrarily fixed $\xi, \eta, R$ and $\sigma$ we obtain for $0<\xi<\eta<R, 0<\sigma<1$ and for each transcendental $B_{\jmath}$

$$
\begin{aligned}
& \int_{\xi}^{\eta}\left\{\log ^{+} \mu\left(r, B_{j}\right)+\frac{\pi \sigma}{\sin \pi \sigma}\left[N\left(r, \infty, B_{j}\right)-\cos \pi \sigma \cdot N\left(r, 0, B_{j}\right)\right]\right\} \frac{d r}{r^{1+\sigma}} \\
\geqq & C_{j}^{\prime} H_{j}(\xi) \xi^{-\sigma}-C_{j}^{\prime \prime}(1-\sigma)^{-1} R^{-\sigma} T\left(2 R, B_{j}\right),
\end{aligned}
$$

where $C_{j}^{\prime}$ and $C_{j}^{\prime \prime}$ are two positive constants. Summing up these inequalities we have for $0<\xi<\eta<R$ and $0<\sigma<1$

$$
\begin{aligned}
& \int_{\xi}^{\eta}\left\{\Sigma \log ^{+} \mu\left(r, B_{j}\right)+\frac{\pi \sigma}{\sin \pi \sigma}\left[\Sigma N\left(r, \infty, B_{j}\right)-\cos \pi \sigma \Sigma N\left(r, 0, B_{j}\right)\right]\right\} \frac{d r}{r^{1+\sigma}} \\
\geqq & \Sigma C_{j}^{\prime} H_{j}(\xi) \xi^{-\sigma}-\Sigma C_{j}^{\prime \prime}(1-\sigma)^{-1} R^{-\sigma} T\left(2 R, B_{j}\right),
\end{aligned}
$$

where each $\Sigma$ is taken over all $j$ such that the $A_{\text {, in }}$ (2.1) are transcendental.

Now we choose $\sigma$ so that $\lambda<\sigma<1$. No matter how large $\xi$ is we can choose the quantity $R=2 \eta$ such that the right side of (5.3) will be positive since each $B_{j}$ 
is of lower order at most $\lambda$ from (5.2) and (3.3). It follows that

$$
\limsup _{r \rightarrow \infty}\left\{\Sigma \log ^{+} \mu\left(r, B_{j}\right)+\frac{\pi \sigma}{\sin \pi \sigma}\left[\Sigma N\left(r, \infty, B_{j}\right)-\cos \pi \sigma \Sigma N\left(r, 0, B_{j}\right)\right]\right\} \geqq 0 .
$$

Thus for an arbitrarily given $\varepsilon>0$ there exists a sequence $\left\{r_{n}\right\}, r_{n} \rightarrow \infty$ as $n \rightarrow \infty$, such that

$$
\Sigma \log ^{+} \mu\left(r_{n}, B_{j}\right) \geqq \frac{\pi \sigma}{\sin \pi \sigma}\left[\cos \pi \sigma \Sigma N\left(r_{n}, 0, B_{j}\right)-\Sigma N\left(r_{n}, \infty, B_{j}\right)\right]-\varepsilon .
$$

From this inequality and (5.2) we deduce

$$
\begin{aligned}
\Sigma \log ^{+} \mu\left(r_{n}, A_{j}\right)+O\left(\log r_{n}\right) \geqq & \frac{\pi \sigma}{\sin \pi \sigma}\left[\cos \pi \sigma \Sigma(1+o(1)) T\left(r_{n}, A_{j}\right)\right. \\
& \left.-\Sigma N\left(r_{n}, \infty, A_{j}\right)+O\left(\log r_{n}\right)\right]-\varepsilon .
\end{aligned}
$$

By dividing both sides of the above inequality by $k T\left(r_{n}, y\right)$ and letting $r_{n}$ tend to infinity in due consideration of (3.2) and (3.3) we have with the arbitrariness of $\varepsilon$

$$
\limsup _{r \rightarrow \infty} \frac{\Sigma \log ^{+} \mu\left(r, A_{j}\right)}{k T(r, y)} \geqq \frac{\pi \sigma}{\sin \pi \sigma}\left[\frac{n}{k} \cos \pi \sigma-n+n \delta(\infty)\right] .
$$

Further we let $\sigma$ tend to $\lambda$. Thus the combination of these with (3.4) yields our theorem.

The proof of our theorem is completed.

\section{REFERENCES}

[1] Gold’Berg, A. A., AND I. V. Ostrovski,, Распределение Эначений Мероморфных Функций. Moscow (1970), 591 pp.

[2] KJellberg, B., On the minimum modulus of entire functions of lower order less than one. Math. Scand. 8 (1960), 189-197.

[3] Nevanlinna, R., Analytic Functions. Springer-Verlag Berlin Heidelberg. New York (1970), $373 \mathrm{pp}$.

[4] Ostrovskil, I. V., On defects of meromorphic functions with lower order less than one. Soviet Math. Dokl. 4 (1963), 587-591.

[5] Ozawa, M., On the minimum modulus of an entıre algebroid function of lower order less than one. Köda1 Math. Sem. Rep. 22 (1970), 166-171.

[6] Selberg, H. L., Algebroide Funktionen und Umkehrfunktionen Abelscher Integrale. Avh. Norske Vid. Akad. Oslo 8 (1934), 1-72.

Department of Mathematics,

Defense Academy, 